SUPPORTING INFORMATION FOR

\title{
Benefits of Iterative Searches of Large Databases to Interpret Large Human Gut Metaproteomic Data Sets
}

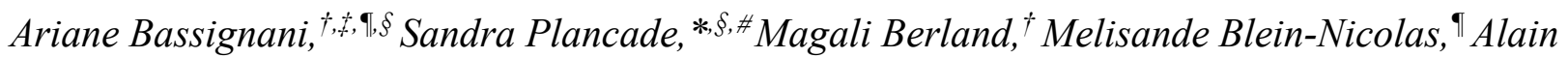

Guillot, ${ }^{\ddagger}$ Didier Chevret, ${ }^{\ddagger}$ Chloé Moritz, ${ }^{\ddagger}$ Sylvie Huet, ${ }^{\S}$ Salwa Rizkalla ${ }^{k, \perp}$ Karine Clément,${ }^{k, \perp}$ Joël Doré, ${ }^{\ddagger}$ Olivier Langella, $* \pi$ and Catherine Juste, $* *$

†Université Paris-Saclay, INRAE, MGP, 78350, Jouy-en-Josas, France; ‘ Université ParisSaclay, INRAE, AgroParisTech, Micalis Institute, 78350, Jouy-en-Josas, France.; "Université Paris-Saclay, INRAE, CNRS, AgroParisTech, GQE - Le Moulon, 91190, Gif-sur-Yvette, France ; ${ }^{\circledR}$ MaIAGE, INRAE, Université Paris-Saclay, 78350 Jouy-en-Josas, France ; \#INRAE,

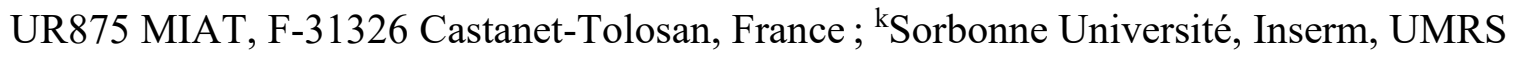
Nutrition et Obésités; approches systémiques, Paris, France ; ${ }^{\perp}$ Assistance Publique Hôpitaux de Paris, Service de Nutrition, CRNH Ile-de-France, Pitié-Salpêtrière Hospital, Paris, France.

*Corresponding authors

*E-mail: sandra.plancade@inrae.fr

*E-mail: olivier.langella@universite-paris-saclay.fr

*E-mail: catherine.juste@inrae.fr 
TABLE OF CONTENT

Supporting Experimental Section S1. Stool Sample Collection and Processing

Supporting Experimental Section S2. Protein Digestion and Peptide Desalting

Supporting Experimental Section S3. LC-MS/MS Analysis

Table S1. Analytical sequence of the samples

Table S2. Summary of the three search strategies

Table S3. Taxonomic diversity allowed by each of the three search strategies

Figure S1. Experimental Design

Figure S2. Overview of the three interrogation methods used in this paper.

Figure S3. Clustering of Proteins using the X!Tandem Grouping Algorithm of

X!TandemPipeline

Figure S4. Plot of the mass delta distributions for the three methods

Figure S5. Species distribution among phyla in the intersection of the three searches (A), or in the additional pool brought by the two-step (B) and the three-step strategy (C)

Figure S6. iPath projection of KO entries highlighted with the three approaches

Figure S7. Distribution of metaproteins as a function of KO entries embedded

Figure S8. If present, the functional diversity of metaproteins is related to the own functional diversity of its component proteins, not to their number

Figure S9. Percentages of genes (A) and metaproteins (B, recall of Figure 4A) assigned to the different phyla within the 48 microbiomes

Figure S10. Example of reproducibility of the positioning of replicates relative to other samples in the cohort, based on relative abundances of all metaproteins

Figure S11. Metaproteins overlapping in all pairs of replicates when singletons are omitted. 


\section{SUPPORTING EXPERIMENTAL SECTION}

\section{Supporting Experimental Section S1: Stool Sample Collection and Processing}

All samples were self-collected as previously detailed ${ }^{18}$. About $1 \mathrm{~g}$ stool aliquots were cut frozen and the microbiota was separated from the fecal matrix by flotation in a preformed Nycodenz continuous gradient according to a variant of the method previously detailed by Juste et al. ${ }^{18}$ Here, we just reduced the size of the gradient. Briefly, stool specimens were supplemented up to $2.82 \mathrm{~g}$ with 1 X PBS $0.03 \%$ w/v Na-deoxycholate, then with $8 \mathrm{ml}$ of Nycodenz $60 \%$, and $6.5 \mathrm{ml}$ of this suspension were loaded below a preformed gradient which has been prepared with $5 \mathrm{ml}$ of a $23 \%$ w/v Nycodenz solution in an Ultra-Clear centrifuge tube $(14.5$ X 95mm, Beckman Instruments, CA, USA). During low-speed ultracentrifugation in a swinging SW 40 Ti rotor (Beckman, 14,567 g, $\left.45 \mathrm{~min}, 4^{\circ} \mathrm{C}\right)$, bacterial cells migrated up to their buoyant density (d 1.110-1.190) while the unwanted fecal matrix sedimented. After washing in cold Tris saline (20 mM Tris, $138 \mathrm{mM} \mathrm{NaCl}$, $2.7 \mathrm{mM} \mathrm{KCl}, 0.03 \% \mathrm{w} / \mathrm{v}$ Na-deoxycholate, $\mathrm{pH} 7.4$ ), the extracted microbiota were frozen in liquid nitrogen then kept at $-80^{\circ} \mathrm{C}$ in $2 \mathrm{~mL}$ screw cap Sarstedt tubes. For bacterial lysis, $1.5 \mathrm{~mL}$ of cold saline Tris-EDTA buffer $(50 \mathrm{mM}$ Tris- $\mathrm{HCl}$, pH 7.8 containing $150 \mathrm{mM} \mathrm{NaCl}$ and $1 \mathrm{mM}$ EDTA, and extemporally supplemented with PMSF at a final concentration of $2 \mathrm{mM}$ and protease inhibitor cocktail (cOmplete ${ }^{\mathrm{TM}}$, EDTA-free Protease Inhibitor Cocktail, ROCHE) at a final concentration of $1.3 \mathrm{X}$ ), was directly added to each frozen bacterial pellet. The pellets were dispersed by vigorous vortexing and sonicated on ice using a $3 \mathrm{~mm}$ diameter probe in short intervals of $10 \mathrm{sec} \mathrm{ON} / 10$ sec OFF, with $20 \%$ amplitude, and for two 5 min periods separated by a 15 min break on ice with periodic vigorous vortexing. Finally, the suspensions were centrifuged at $5000 \mathrm{~g}$ for $30 \mathrm{~min}$ at $4^{\circ} \mathrm{C}$ to remove unbroken cells and large cellular debris. The supernatant were ultracentrifuged in a swinging rotor (SW $55 \mathrm{Ti}, \mathrm{Beckman}$ ) at $220,000 \mathrm{~g}$ for $30 \mathrm{~min}$ at $4^{\circ} \mathrm{C}$ to separate cell envelopes (pellet) and cytosolic fractions (supernatant). 


\section{Supporting Experimental Section S2: Protein Digestion and Peptide Desalting}

Cell envelope-enriched pelleted in the ultracentrifuge tubes were resuspended in a minimal volume $(40 \mu \mathrm{L})$ of $50 \mathrm{mM} \mathrm{NH}_{4} \mathrm{HCO}_{3}$ containing $0.2 \%$ ProteaseMAX ${ }^{\mathrm{TM}}$ surfactant (ref Promega V2072), using a Microman pipette fitted with a $50 \mu \mathrm{L}$ capillary piston, and then sonicated and vortexed until homogeneous suspensions were obtained. The suspensions were transferred in acetonecompatible $2 \mathrm{~mL}$ Protein LoBind tubes (ref Eppendorf 0030108132), added with $60 \mu \mathrm{L}$ of ultracentrifuge tube rinsing $\mathrm{NH}_{4} \mathrm{HCO}_{3}(50 \mathrm{mM}$, detergent-free), and then precipitated on ice with four volumes of pure ice-cold $\left(-20^{\circ} \mathrm{C}\right)$ acetone. The suspension was chilled down to $-20^{\circ} \mathrm{C}$ with vigorous vortexing at $10 \mathrm{~min}$ intervals over the first $30 \mathrm{~min}$, and then left at $-20^{\circ} \mathrm{C}$ overnight. The next morning, suspensions were transferred for $10 \mathrm{~min}$ at $-80^{\circ} \mathrm{C}$ before being centrifuged at 16,000 $\mathrm{g}, 4^{\circ} \mathrm{C}$ for $10 \mathrm{~min}$. Supernatants were decanted and the protein pellets were washed twice with icecold $\left(-20^{\circ} \mathrm{C}\right)$ acetone $80 \%$ in MilliQ water. Protein concentration was determined before the second acetone wash decanting, using the 2D-Quant kit (ref GE Healthcare 80-6483-5, shortcircuiting precipitant/coprecipitant use of the kit as in our case proteins were precipitated by acetone). Aliquots equivalent to $50 \mu \mathrm{g}$ protein were frozen in liquid nitrogen then kept at $-80^{\circ} \mathrm{C}$. Reduction, alkylation, and liquid digestion in the presence of Trypsin Gold (ref Promega V5280) in a trypsin-to-protein ratio of 1:50 (w:w), and of ProteaseMax ${ }^{\mathrm{TM}}$ as the surfactant were essentially as recommended in the Promega technical bulletin (https://www.promega.com//media/files/resources/protocols/technical-bulletins/101/proteasemax-surfactant-trypsinenhancer.pdf?la=en). Finally, peptide mixtures were desalted on Sep-Pak tC18 Plus Short cartridges (ref Waters WAT036810) with 35\% acetonitrile then 50\% in the final elution steps. The frozen eluates were lyophilized on a Thermo Scientific Savant SpeedVac vacuum concentrator. 


\section{Supporting Experimental Section S3: LC-MS/MS Analysis}

HPLC was performed on an Eksigent NanoLC-Ultra system (Eksigent, Les Ulis, France). Trypsic digestion products $(7 \mu \mathrm{g})$ were loaded, concentrated and desalted on a precolumn cartridge (BIOSPHERE C18, $5 \mu \mathrm{m}$ particle size; column: $100 \mu \mathrm{m}$ i.d., $2 \mathrm{~cm}$ length; NanoSeparations Nieuwkoop, The Netherlands) with $0.1 \% \mathrm{HCOOH}$ at $7.5 \mu \mathrm{L} \cdot \mathrm{min}^{-1}$ for $3 \mathrm{~min}$. The precolumn cartridge was connected to the separating column (Acclaim PepMap100, $3 \mu \mathrm{m}, 100 \AA, 75 \mu \mathrm{m}$ i.d. $50 \mathrm{~cm}$ length, ThermoFisher Scientfic) and the peptides were eluted with a non-linear gradient from 5 to $35 \% \mathrm{ACN}$ in $0.1 \% \mathrm{HCOOH}$ for $180 \mathrm{~min}$ at $300 \mathrm{~nL} \cdot \mathrm{min}^{-1}$. On line analysis of peptides was performed with a Qexactive mass spectrometer (ThermoFisher Scientific), using a nanoelectrospray ion source (noncoated capillary probe, 10 i.d.; New Objective, Woburn, MA, USA). Peptide ions were analysed using Xcalibur 2.1 with the following data-dependent acquisition steps: (1) full MS scan (mass-tocharge ratio $(\mathrm{m} / \mathrm{z}) 300$ to 1,400 , resolution 70,000) and (2) MS/MS (normalized collision energy = $30 \%$, resolution 17,500). Step 2 was repeated for the 12 major ions detected in step 1. Dynamic exclusion was set to $60 \mathrm{sec}$. 


\section{Supporting Tables}

Table S1. Analytical sequence of the samples

\begin{tabular}{|c|c|c|c|c|c|c|c|c|c|}
\hline Date of injection & $\begin{array}{l}\text { Time of } \\
\text { injection }\end{array}$ & Subject & gradient & injection & $\begin{array}{l}\text { LC.MS.MS } \\
\text { series }\end{array}$ & $\begin{array}{l}\text { rank within the } \\
\text { series }\end{array}$ & $\begin{array}{l}\text { gradient } \\
\text { operator }\end{array}$ & $\begin{array}{l}\text { LC.MS.MS } \\
\text { operator }\end{array}$ & $m \times X M L$ file \\
\hline 2014-07-17 & 09:53:29 & s32 & A & 1 & 1 & 2 & $\mathrm{CF}$ & AL & 2014_07_16_ENV10_01.mzXML \\
\hline 2014-07-17 & 17:26:30 & S07 & A & 1 & 1 & 3 & $\mathrm{CA}$ & AL & 2014_07_16_ENV10_02.mzXML \\
\hline 2014-07-18 & 04:48:26 & s08 & A & 1 & 1 & 6 & $\mathrm{CA}$ & $\mathrm{AL}$ & 2014_07_16_Obomics_S15D0_01.mzXML \\
\hline 2014-07-18 & $16: 10: 18$ & $\mathrm{~S} 15$ & A & 1 & 1 & 9 & $\mathrm{CA}$ & $\mathrm{AL}$ & 2014_07_16_Obomics_S21D0_01.mzXML \\
\hline 2014-07-19 & 03:32:09 & $\mathrm{s} 21$ & A & 1 & 1 & 12 & $\mathrm{CA}$ & AL & 2014_07_16_Obomics_S22D0_01.mzXML \\
\hline 2014-07-19 & 14:54:06 & $\mathrm{S} 22$ & A & 1 & 1 & 15 & $\mathrm{CA}$ & $\mathrm{AL}$ & 2014_07_16_Obomics_S26D0_01.mzXML \\
\hline 2014-07-20 & 02:16:00 & $\mathrm{s} 26$ & A & 1 & 1 & 18 & $\mathrm{CA}$ & $\mathrm{AL}$ & 2014_07_16_Obomics_S35D0_01.mzXML \\
\hline 2014-07-20 & 13:37:57 & S35 & A & 1 & 1 & 21 & CC & AL & 2014_07_16_Obomics_S7D0_01.mzXML \\
\hline 2014-07-21 & 00:59:51 & s32 & A & 2 & 1 & 24 & $\mathrm{CF}$ & AL & 2014_07_16_Obomics_S8D0_01.mzXML \\
\hline 2014-07-23 & 20:17:15 & s32 & A & 3 & 1 & 25 & $\mathrm{CF}$ & AL & 2014_07_23_ENV10_03.mzXML \\
\hline 2014-07-24 & 10:10:48 & $\mathrm{S} 38$ & A & 1 & 1 & 28 & $\mathrm{CC}$ & AL & 2014_07_23_ENV10_04.mzXML \\
\hline 2014-07-24 & $21: 32: 46$ & S48 & A & 1 & 1 & 31 & $\mathrm{CA}$ & $\mathrm{AL}$ & 2014_07_23_Obomics_S38D0_01.mzXML \\
\hline 2014-07-25 & 08:54:41 & $\mathrm{S} 49$ & A & 1 & 1 & 34 & $\mathrm{CA}$ & $\mathrm{AL}$ & 2014_07_23_Obomics_S48D0_01.mzXML \\
\hline 2014-07-26 & 07:38:37 & s32 & A & 4 & 1 & 39 & $\mathrm{CF}$ & AL & 2014_07_23_Obomics_S49D0_01.mzXML \\
\hline 2014-09-30 & 11:32:37 & $\mathrm{S} 03$ & A & 1 & 2 & 1 & $\mathrm{CC}$ & $\mathrm{AL}$ & 2014_09_29_ENV10_05.mzXML \\
\hline 2014-09-30 & 22:54:40 & s04 & A & 1 & 2 & 4 & $\mathrm{CC}$ & $\mathrm{AL}$ & 2014_09_30_Obomics_S03D0_01.mzXML \\
\hline 2014-09-30 & 00:34:10 & s32 & A & 5 & 2 & 5 & $\mathrm{CF}$ & AL & 2014_09_30_Obomics_S04D0_01.mzXML \\
\hline 2014-10-01 & 10:16:39 & $\mathrm{s} 11$ & A & 1 & 2 & 8 & $\mathrm{CA}$ & $\mathrm{AL}$ & 2014_09_30_Obomics_S11D0_02.mzXML \\
\hline 2014-10-06 & 14:13:00 & S32 & A & 6 & 2 & 11 & CF & AL & 2014_10_06_ENV10_06.mzXML \\
\hline 2014-10-06 & $22: 10: 18$ & $\mathrm{~S} 14$ & A & 1 & 2 & 12 & $\mathrm{CA}$ & $\mathrm{AL}$ & 2014_10_06_Obomics_S14D0_01.mzXML \\
\hline 2014-10-07 & $16: 24: 33$ & $\mathrm{~S} 25$ & A & 1 & 2 & 15 & $\mathrm{CA}$ & AL & 2014_10_07_Obomics_S25D0_01.mzXML \\
\hline $2014-10-08$ & 03:46:32 & $\mathrm{S} 30$ & A & 1 & 2 & 18 & $\mathrm{CA}$ & AL & 2014_10_07_Obomics_S30D0_01.mzXML \\
\hline 2014-10-08 & 15:08:27 & $\mathrm{S} 31$ & A & 1 & 2 & 21 & $\mathrm{CA}$ & AL & 2014_10_07_Obomics_S31D0_01.mzXML \\
\hline 2014-10-09 & 02:30:26 & $\mathrm{S} 33$ & A & 1 & 2 & 24 & $\mathrm{CC}$ & $\mathrm{AL}$ & 2014_10_07_Obomics_S33D0_01.mzXML \\
\hline 2014-10-09 & 13:52:28 & $\mathrm{s} 36$ & A & 1 & 2 & 27 & CC & $\mathrm{AL}$ & 2014_10_07_Obomics_S36D0_01.mzXML \\
\hline 2014-10-10 & 01:14:20 & s37 & A & 1 & 2 & 30 & CC & $\mathrm{AL}$ & 2014_10_07_Obomics_S37D0_01.mzXML \\
\hline 2014-10-13 & 13:06:37 & s32 & A & 7 & 2 & 33 & $\mathrm{CF}$ & AL & 2014_10_13_ENV10_07.mzXML \\
\hline 2014-10-13 & 17:37:37 & S39 & A & 1 & 2 & 34 & $\mathrm{CC}$ & $\mathrm{AL}$ & 2014_10_13_ENV10_08.mzXML \\
\hline 2014-10-14 & 04:59:38 & $\mathrm{S} 40$ & A & 1 & 2 & 37 & $\mathrm{CC}$ & AL & 2014_10_13_ENV10_08_141018202350.mzXML \\
\hline 2014-10-15 & 03:43:54 & $\mathrm{S} 42$ & A & 1 & 2 & 43 & $\mathrm{CC}$ & AL & 2014_10_13_Obomics_S39D0_01.mzXML \\
\hline 2014-10-15 & 15:06:04 & $\mathrm{S} 43$ & A & 1 & 2 & 46 & $\mathrm{CA}$ & AL & 2014_10_13_Obomics_S40D0_01.mzXML \\
\hline 2014-10-16 & 02:28:20 & S46 & A & 1 & 2 & 49 & $\mathrm{CA}$ & $\mathrm{AL}$ & 2014_10_13_Obomics_S42D0_01.mzXML \\
\hline 2014-10-16 & 18:19:31 & S32 & A & 9 & 2 & 52 & $\mathrm{CF}$ & AL & 2014_10_13_Obomics_S43D0_01.mzXML \\
\hline 2014-10-16 & 22:50:35 & S47 & A & 1 & 2 & 53 & $\mathrm{CA}$ & $\mathrm{AL}$ & 2014_10_13_Obomics_S46D0_01.mzXML \\
\hline 2014-10-17 & 10:13:31 & S51 & A & 1 & 2 & 56 & $\mathrm{cc}$ & AL & 2014_10_13_Obomics_S47D0_01.mzXML \\
\hline 2014-10-17 & $21: 36: 54$ & S52 & A & 1 & 2 & 59 & $\mathrm{CC}$ & $\mathrm{AL}$ & 2014_10_13_Obomics_S51D0_01.mzXML \\
\hline 2014-10-18 & 08:59:47 & S53 & A & 1 & 2 & 61 & $\mathrm{CC}$ & $\mathrm{AL}$ & 2014_10_13_Obomics_S52D0_01.mzXML \\
\hline 2014-10-18 & 20:23:50 & s32 & A & 8 & 2 & 64 & CF & AL & 2014_10_13_Obomics_S53D0_01.mzXML \\
\hline 2015-11-13 & 23:10:02 & s32 & B & 2 & 3 & 1 & CA & DI & 20151112_S1_D0.mzXML \\
\hline 2015-11-14 & 02:35:38 & s32 & c & 1 & 3 & 2 & cc & DI & 20151112_S10_D0_A.mzXML \\
\hline 2015-11-15 & 00:14:16 & s01 & A & 1 & 3 & 8 & $\mathrm{CC}$ & $\mathrm{DI}$ & 20151112_S13_D0_A.mzXML \\
\hline 2015-11-15 & $11: 36: 21$ & S02 & A & 1 & 3 & 11 & $\mathrm{CC}$ & DI & 20151112_S16_D0_.mzXML \\
\hline 2015-11-16 & $16: 28: 21$ & s32 & B & 1 & 3 & 14 & CA & DI & 20151112_S17_DOreinj2.mzXML \\
\hline 2015-11-16 & 20:59:21 & s06 & A & 1 & 3 & 15 & $\mathrm{CA}$ & $\mathrm{DI}$ & 20151112_S19_D0_reinj.mzXML \\
\hline 2015-11-17 & 23:08:44 & sog & A & 1 & 3 & 20 & $\mathrm{cc}$ & $\mathrm{DI}$ & 20151112_S2_D0.mzXML \\
\hline $2015-11-18$ & 10:30:46 & $\mathrm{S} 10$ & A & 1 & 3 & 23 & $\mathrm{CA}$ & $\mathrm{DI}$ & 20151112_S20_DOreinj.mzXML \\
\hline $2015-11-19$ & 01:18:24 & $\mathrm{S} 13$ & A & 1 & 3 & 27 & $\mathrm{CC}$ & $\mathrm{DI}$ & 20151112_S23_Do_Areinj.mzXML \\
\hline 2015-11-19 & 16:05:57 & s32 & B & 3 & 3 & 31 & CA & DI & 20151112_S27_DOreinj.mzXML \\
\hline 2015-11-19 & 20:36:59 & $\mathrm{S} 16$ & A & 1 & 3 & 32 & $\mathrm{CA}$ & $\mathrm{DI}$ & 20151112_S28_DOreinj.mzXML \\
\hline 2015-11-26 & 14:18:18 & S32 & c & 2 & 3 & 38 & $\mathrm{cc}$ & DI & 20151112_S29_D0reinj2.mzXML \\
\hline 2015-11-27 & 13:02:27 & S19 & A & 1 & 3 & 44 & $\mathrm{CA}$ & $\mathrm{DI}$ & 20151112_S32_DO_A_151116162821.mzXML \\
\hline 2015-11-28 & 00:24:35 & $\mathrm{s} 20$ & A & 1 & 3 & 47 & $\mathrm{CA}$ & $\mathrm{DI}$ & 20151112_S32_DO_A3.mzXML \\
\hline 2015-11-28 & 11:46:39 & $\mathrm{s} 23$ & A & 1 & 3 & 50 & $\mathrm{CA}$ & $\mathrm{DI}$ & 20151112_S32_DO_A.mzXML \\
\hline 2015-11-29 & 02:34:15 & S27 & A & 1 & 3 & 54 & $\mathrm{CA}$ & $\mathrm{DI}$ & 20151112_S32_DO_B2_reinj2.mzXML \\
\hline $2015-11-29$ & 21:52:49 & S17 & A & 3 & 3 & 58 & $\mathrm{CA}$ & $\mathrm{DI}$ & 20151112_S32_DO_B.mzXML \\
\hline 2015-11-30 & 02:23:48 & $\mathrm{S} 28$ & A & 1 & 3 & 59 & $\mathrm{CA}$ & $\mathrm{DI}$ & 20151112_S34_D0.mzXML \\
\hline 2015-11-30 & $13: 45: 53$ & $\mathrm{~s} 29$ & A & 1 & 3 & 62 & $\mathrm{CA}$ & $\mathrm{DI}$ & 20151112_S5_D0_reinj.mzXML \\
\hline 2015-12-01 & 01:07:52 & S 34 & A & 1 & 3 & 65 & $\mathrm{CC}$ & $\mathrm{DI}$ & 20151112_S6_D0.mzXML \\
\hline 2015-12-01 & $12: 29: 49$ & S44 & A & 1 & 3 & 68 & $\mathrm{CA}$ & $\mathrm{DI}$ & 20151112_S9_D0.mzXML \\
\hline 2015-12-02 & $06: 42: 59$ & $\mathrm{~S} 45$ & A & 1 & 3 & 73 & $\mathrm{CA}$ & $\mathrm{DI}$ & 2015112_S44_DOA.mzXML \\
\hline 2015-12-03 & 02:01:45 & $\mathrm{s} 05$ & A & 1 & 3 & 78 & $\mathrm{CC}$ & $\mathrm{DI}$ & 2015112_S45_DOA.mzXML \\
\hline
\end{tabular}


Table S2. Summary of the three search strategies

\begin{tabular}{|c|c|c|c|c|c|c|}
\hline & Classical & \multicolumn{2}{|c|}{ 2-step iterative } & \multicolumn{3}{|c|}{ 3-step iterative } \\
\hline Number of RAW files & \multicolumn{5}{|c|}{48} & \multirow[b]{3}{*}{ Step 3} \\
\hline Total number of MS/MS spectra & \multicolumn{5}{|c|}{1370929} & \\
\hline $\begin{array}{l}\text { Number of sequences (amino acids) } \\
\text { in the target database }\end{array}$ & Step 1 & Step 1 & Step 2 & Step 1 & Step 2 & \\
\hline Microbial sequences & $\begin{array}{c}9879896 \\
(2470939150)\end{array}$ & $\begin{array}{c}9879896 \\
(2470939150)\end{array}$ & $\begin{array}{c}1396170 \\
(553006294)\end{array}$ & $\begin{array}{c}9879896 \\
(2470939150)\end{array}$ & $\begin{array}{c}130125 \pm 25686 \\
(55261845 \pm 10330967)\end{array}$ & $\begin{array}{c}541324 \\
(224295374)\end{array}$ \\
\hline Human sequences & \multicolumn{6}{|c|}{$\begin{array}{c}195418 \\
(67022290)\end{array}$} \\
\hline Contaminant sequences & \multicolumn{6}{|c|}{$\begin{array}{c}58 \\
(26407)\end{array}$} \\
\hline Final percentage of interpreted spectra & 30.9 & \multicolumn{2}{|c|}{33.6} & \multicolumn{3}{|c|}{36.1} \\
\hline
\end{tabular}

Table S3. Taxonomic diversity allowed by each of the three search strategies

\begin{tabular}{|c|c|c|c|}
\hline & Classical & 2-step iterative & 3-step iterative \\
\hline Number of metaproteins & 23131 & 25642 & 28347 \\
\hline Number of HUMAN metaproteins & 608 & 676 & 716 \\
\hline Number of MICROBIAL metaproteins & 22521 & 24964 & 27630 \\
\hline Number of metaproteins containing a mix of histones and microbial proteins & 2 & 2 & 1 \\
\hline Number of species & 2210 & 2376 & 2538 \\
\hline Number of genera & 362 & 380 & 412 \\
\hline Number of families & 155 & 163 & 178 \\
\hline Number of orders & 96 & 102 & 110 \\
\hline Number of classes & 57 & 60 & 62 \\
\hline Number of phyla & 29 & 30 & 32 \\
\hline Number of superkingdoms & 4 & 4 & 4 \\
\hline Percentage of microbial metaproteins with a unique annotation at the species level & 87,75 & 88,00 & 88,42 \\
\hline Percentage of microbial metaproteins with a unique annotation at the genus level & 93,41 & 93,50 & 93,76 \\
\hline Percentage of microbial metaproteins with a unique annotation at thefamily level & 94,63 & 94,71 & 94,93 \\
\hline Percentage of microbial metaproteins with a unique annotation at the class level & 97,78 & 97,76 & 97,89 \\
\hline Percentage of microbial metaproteins with a unique annotation at the phylum level & 98,61 & 98,64 & 98,70 \\
\hline Percentage of microbial metaproteins with a unique annotation at the superkingdom level & 98,95 & 98,98 & 99,00 \\
\hline
\end{tabular}




\section{Supporting Figures}

A

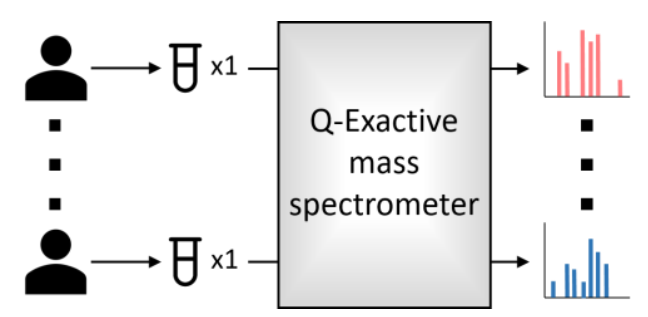

B

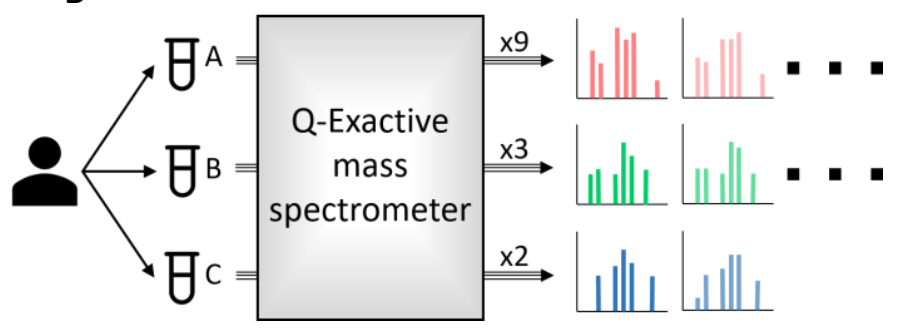

Figure S1. Experimental Design. (A) 47 stool samples were each prepared and injected only once. (B) One stool sample was prepared in triplicates (A, B and C), which were injected nine, three and two times, respectively. 
A

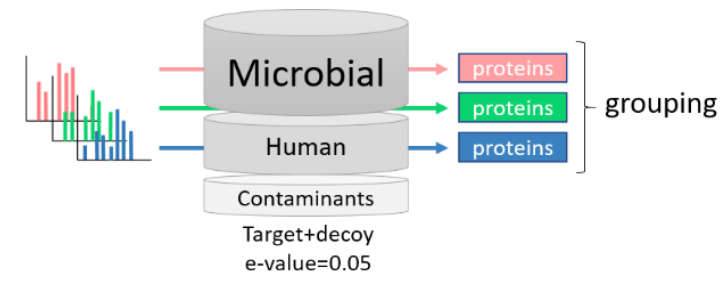

B

(1)
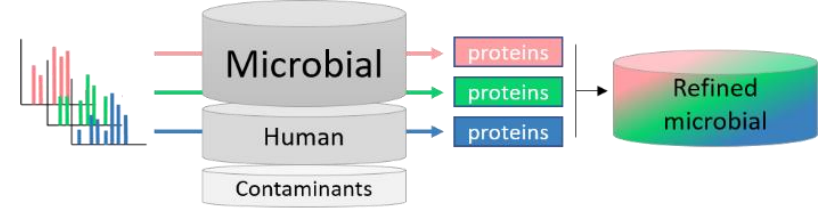

(2)

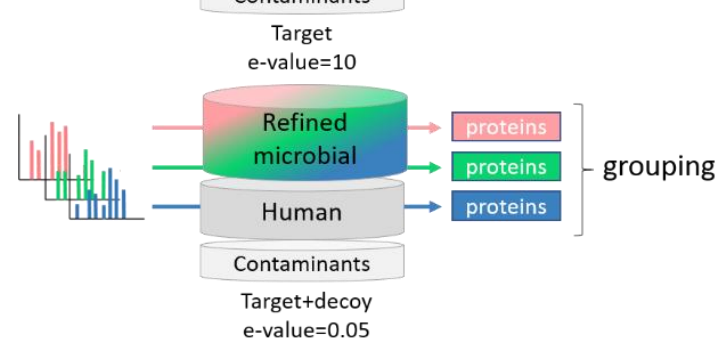

C
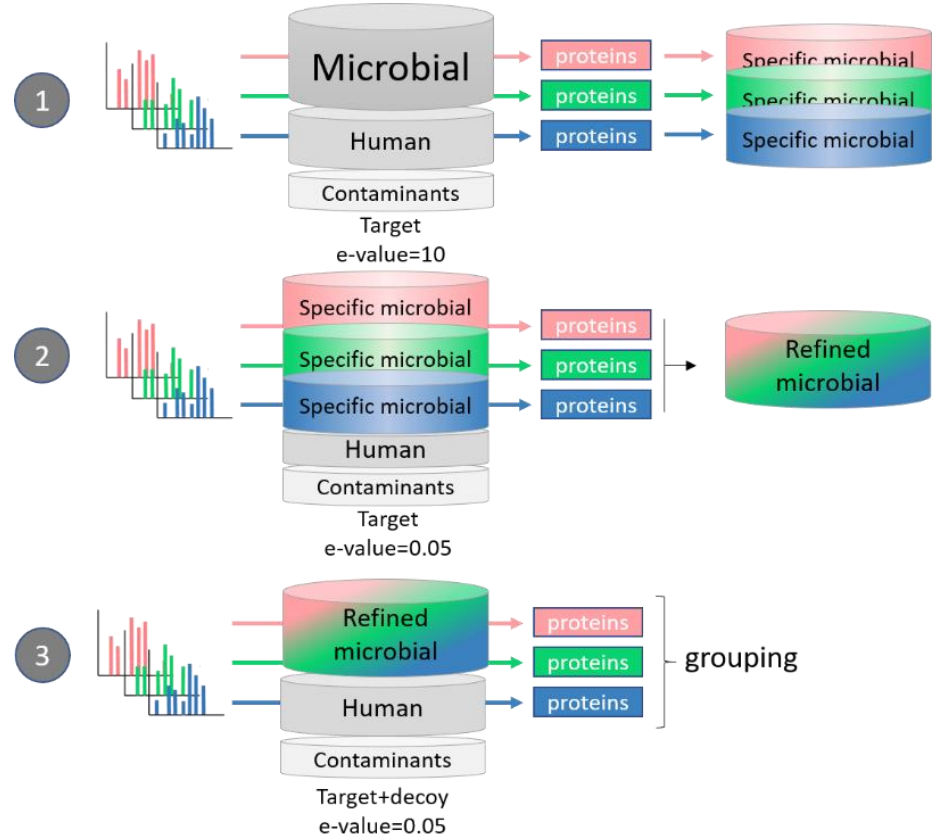

Figure S2. Overview of the three interrogation methods used in this paper. (A) Classical one-step interrogation. (B) Two-step iterative interrogation. (C) Three-step iterative interrogation. 

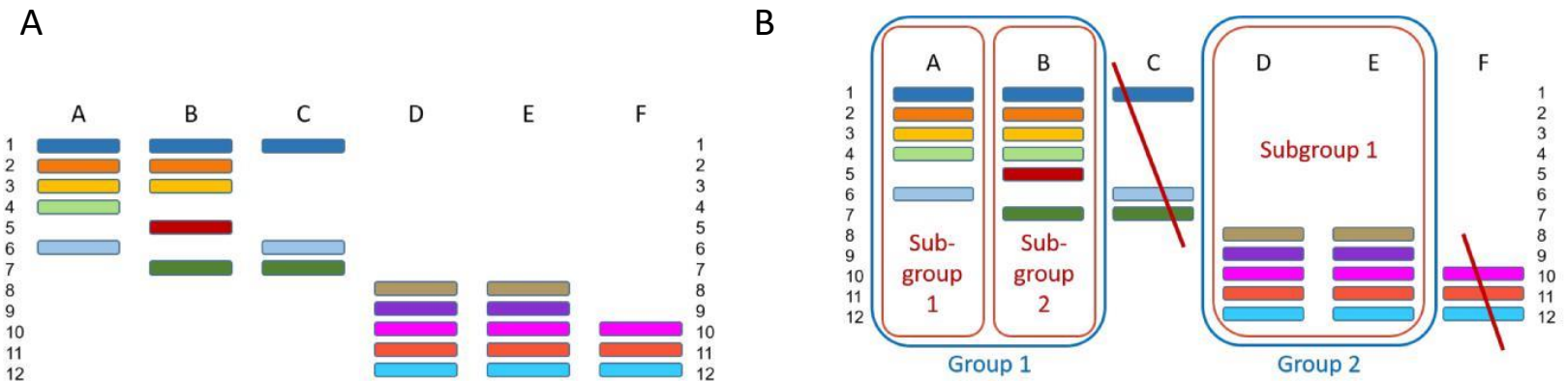

\begin{tabular}{|c|c|c|c|c|c|c|c|c|c|c|}
\hline Group ID & Subgroup ID & Protein ID & accession & description & Number of proteins & 20190111_44 & 20190111 34 & $420190111 \_28$ & 20190111 26 & 520190111440 \\
\hline a1 & a1.a1 & a1.a1.a1 & Sp|P05164|PERM & Myeloperoxidase OS=Homo sapi & 1 & 305 & 339 & 124 & 167 & 99 \\
\hline a1 & a1.a2 & a1.a2.a1 & Sp|P11678|PERE_t & Eosinophil peroxidase OS=Homo & 1 & 19 & 24 & 15 & 25 & 17 \\
\hline a2 & a2.a1 & a2.a1.a1 & $\operatorname{tr}|0.53 G 76| 053 G 7$ & 71 Beta actin variant (Fragment) OS & 1 & 0 & 0 & 0 & 0 & 0 \\
\hline a2 & a2.a2 & a2.a2.a1 & $\operatorname{tr}|B 4 D W 52| B 4 D W$ & V. cDNA FU 55253 , highly similar to. & 1 & 0 & 0 & 0 & 0 & 0 \\
\hline a2 & a2.a3 & a2.a3.a1 & $\operatorname{tr}|\mathrm{B} 4 \mathrm{E} 3 \mathrm{~A} 4| \mathrm{B} 4 \mathrm{E} 3 \mathrm{~A} 4$ & 4 CDNA FU 57283 , highly similar to. & 1 & 2 & 3 & 0 & 4 & 0 \\
\hline a2 & a2.a4 & a2.a4.a1 & $\operatorname{tr}\left|\mathrm{B} 3 \mathrm{KW} \mathrm{W}_{3}\right| \mathrm{B} 3 \mathrm{KW}$ & V cDNA FU 43573 fis, clone RECTM2 & 1 & 0 & 0 & 0 & 0 & 0 \\
\hline a2 & a2.a5 & a2.a5.a1 & sp|P68032|ACTC_ & IActin, alpha cardiac muscle 1 OS: & 1 & 13 & 13 & 10 & 12 & 11 \\
\hline a2 & a2.a6 & a2.a6.a1 & $\operatorname{tr}|B 3 K U D 3| B 3 K U D$ & D cDNA FU 39583 fis, clone SKMUSZ & 1 & 0 & 0 & 3 & 0 & 3 \\
\hline a2 & a2.a7 & a2.a7.a1 & Sp|Q562R1|ACTBL & L Beta-actin-like protein $20 \mathrm{OS}=\mathrm{Hon}$ & 2 & 0 & 0 & 0 & 1 & 0 \\
\hline \multicolumn{11}{|l|}{$\cdots$} \\
\hline d1161 & d1161.a1 & d1161.a1.a1 & $2889179|2786667|$ & | organisms|Bacteria| Firmicutes| & 1 & 0 & 0 & 10 & 0 & 15 \\
\hline d1162 & d1162.a1 & d1162.a1.a1 & $1631095|1623476|$ & | organisms|Bacteria| Firmicutes| & 1 & 0 & 0 & 0 & 0 & 0 \\
\hline d1162 & d1162.a2 & d1162.a2.a1 & $2703683|2608448|$ & | organisms|Bacteria|Firmicutes| & 1 & 0 & 0 & 1 & 1 & 0 \\
\hline d1162 & d1162.a3 & d1162.a3.a1 & $1470833|1464073|$ & | organisms|Bacteria| Firmicutes| & 1 & 0 & 0 & 0 & 0 & 0 \\
\hline d1162 & d1162.a4 & d1162.a4.a1 & $2563197|2482929|$ & | organisms|Bacteria|Firmicutes| & 1 & 0 & 0 & 0 & 0 & 0 \\
\hline d1162 & d1162.a5 & d1162.a5.a1 & $2723227|2626799|$ & | organisms|Bacteria|Firmicutes| & 1 & 0 & 0 & 0 & 0 & 0 \\
\hline d1162 & d1162.a6 & d1162.a6.a1 & $2437718|2368006|$ & | organisms|Bacteria|Firmicutes| & 1 & 0 & 0 & 0 & 0 & 0 \\
\hline d1162 & d1162.a7 & d1162.a7.a1 & $2900573|2797879|$ & | organisms|Bacteria|Actinobacte & 1 & 0 & 0 & 0 & 2 & 0 \\
\hline d1162 & d1162.a8 & d1162.a8.a1 & $143693|143175| G L$ & Lorganisms|Bacteria|Firmicutes| & 1 & 0 & 0 & 0 & 0 & 0 \\
\hline d1162 & d1162.a9 & d1162.a9.a1 & $481978|480400| \mathrm{GL}$ & Lorganisms|Bacteria|NA|NA|NA & 1 & 0 & 0 & 0 & 0 & 0 \\
\hline d1162 & d1162.b10 & d1162.b10.a1 & $2435840|2366199|$ & | organisms|Bacteria|Firmicutes| & 1 & 0 & 0 & 0 & 1 & 0 \\
\hline d1162 & d1162.b11 & d1162.b11.a1 & $2116409|2090057|$ & | organisms|Bacteria|Firmicutes| & 1 & 0 & 0 & 0 & 0 & 0 \\
\hline d1162 & d1162.b12 & d1162.b12.a1 & $1100267|1095485|$ & | organisms|Bacteria|Firmicutes| & 2 & 0 & 0 & 0 & 0 & 0 \\
\hline d1163 & d1163.a1 & d1163.a1.a1 & Sp|Q9BS26|TXND & 4 Thioredoxin domain-containing & 1 & 4 & 5 & 0 & 4 & 0 \\
\hline
\end{tabular}

Figure S3. Grouping of proteins using the X!Tandem Grouping Algorithm of X!TandemPipeline. (A) Peptides (colored bands 1-12) and proteins (A-F) identified by LC-MS/MS. (B) The clustering is to generate a parsimonious list of peptides and proteins identified. Here, $\mathrm{C}$ and $\mathrm{F}$ are removed because their presence is not confirmed by the presence of specific peptides. A and B define two different subgroups because they each have at least one specific peptide (pep 4 and pep 6 for A, pep 5 and pep 7 for B). A and B also share three peptides (pep 1-3), so that they are clustered within the same group. D and E share all their peptides so that they are clustered within the same subgroup, but they share no peptide with A and B, so that they are clustered in a different group. Only subgroup entities were used in the present paper, and referred as to 'metaproteins'. (C) Extract of the output of $\mathrm{X}$ !TandemPipeline after clustering of proteins. Spectral counts for each metaprotein (in rows) and each sample included in the experiment (in columns) are given from column 7. 


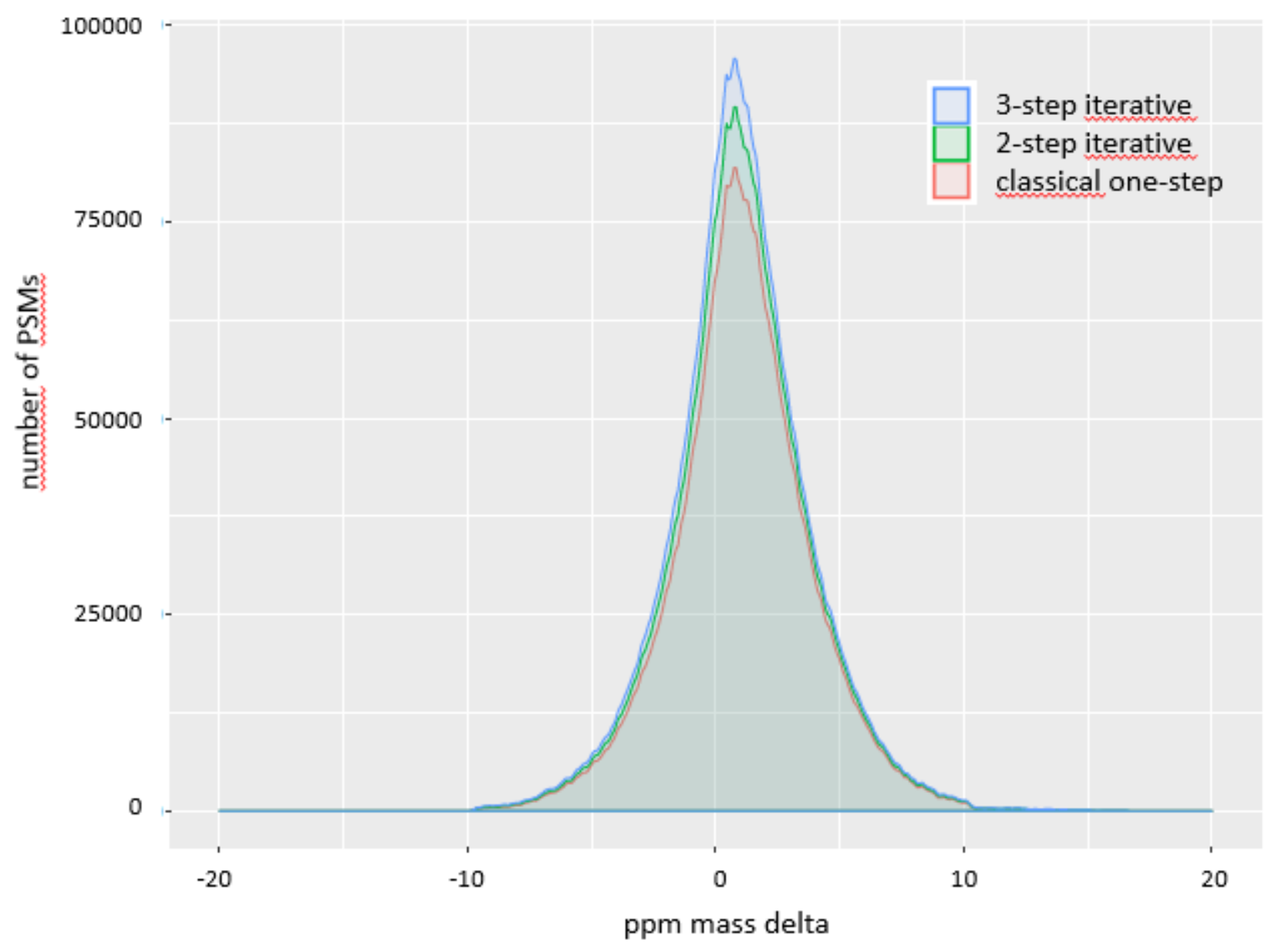

Figure S4. Distribution of the mass delta values (for each PSM, the peptide's calculated mass minus its measured mass in ppm) for the whole dataset (48 samples), and for each method used. Identifications with the three methods follow the same Gaussian distribution, which means that false positives were just as well controlled in the iterative and the classical methods. 


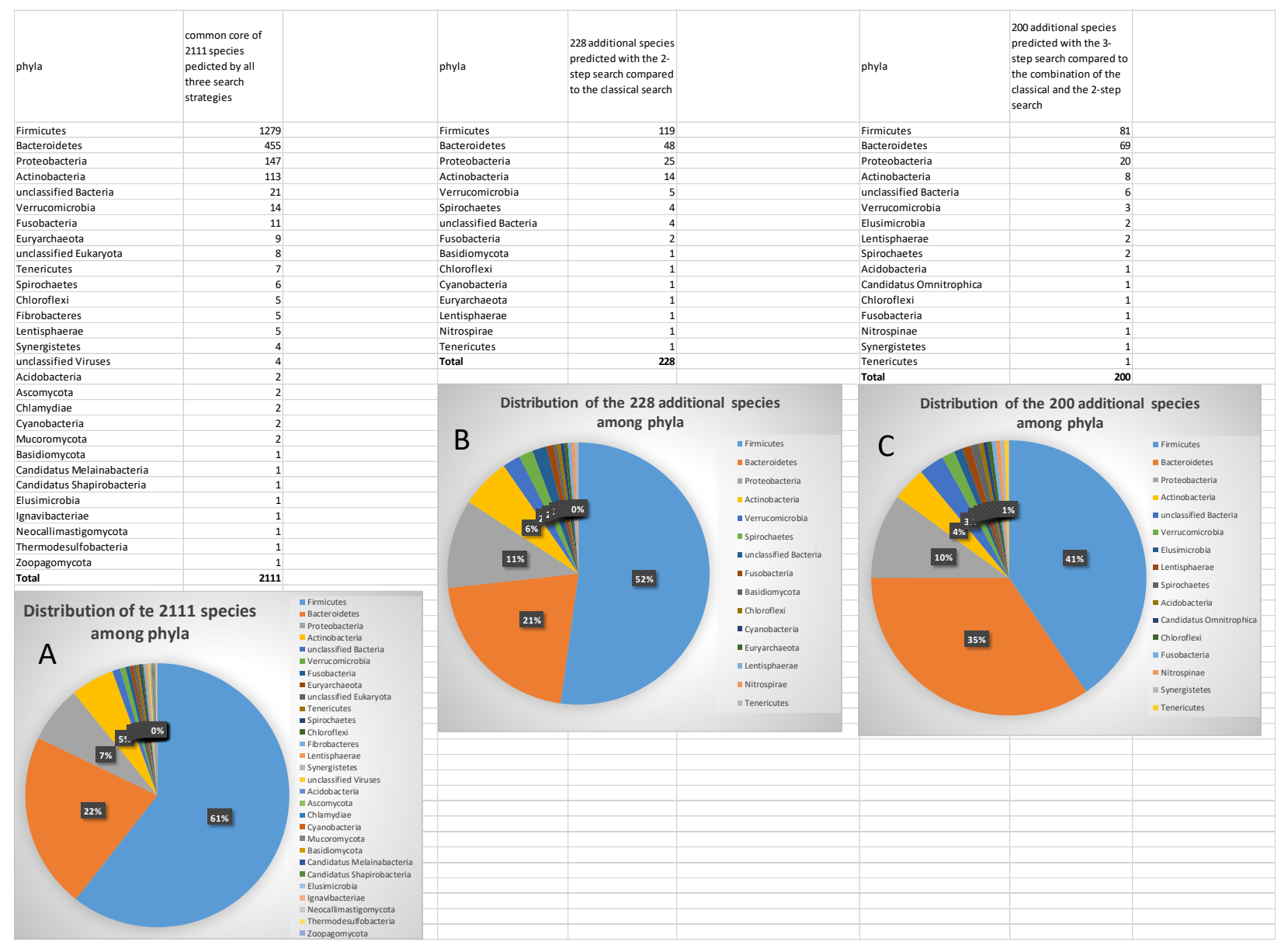

Figure S5. Species distribution among phyla in the intersection of the three searches (A), or in the additional pool brought by the two-step (B) and the three-step strategy (C). 


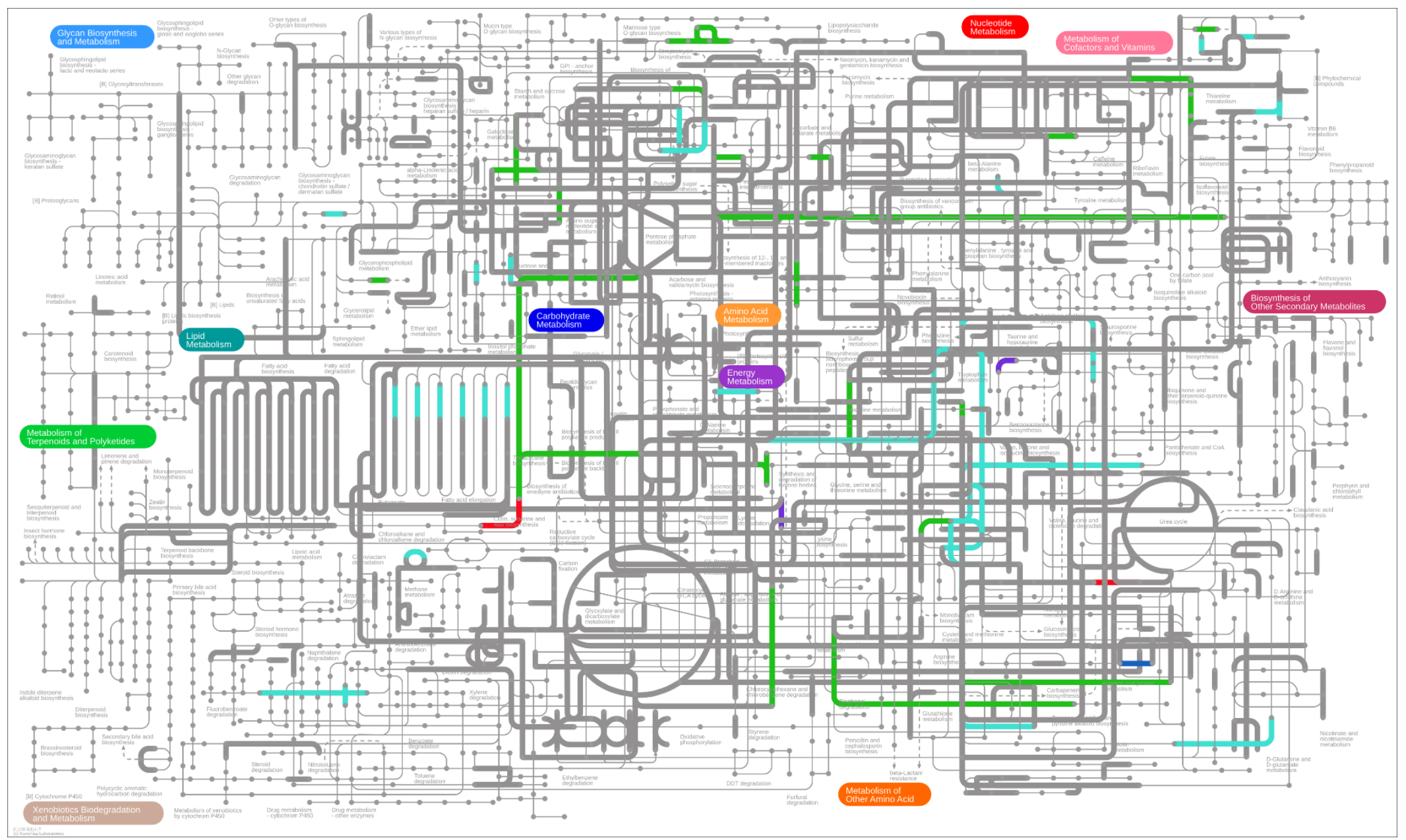

Figure S6. iPath projection of KO entries highlighted with the three approaches: $(-)$ intersection of all three approaches; $(-)$ intersection of supplemental KO entries with the two-step and the three-step iterative strategies; $(-)$ supplemental KO entries specific to the three-step iterative approach. Other minor specificities (red and blue for KO entries specific to the classical and the two-step iterative approach, respectively, and violet for intersection of both) can be visualized by zooming. 

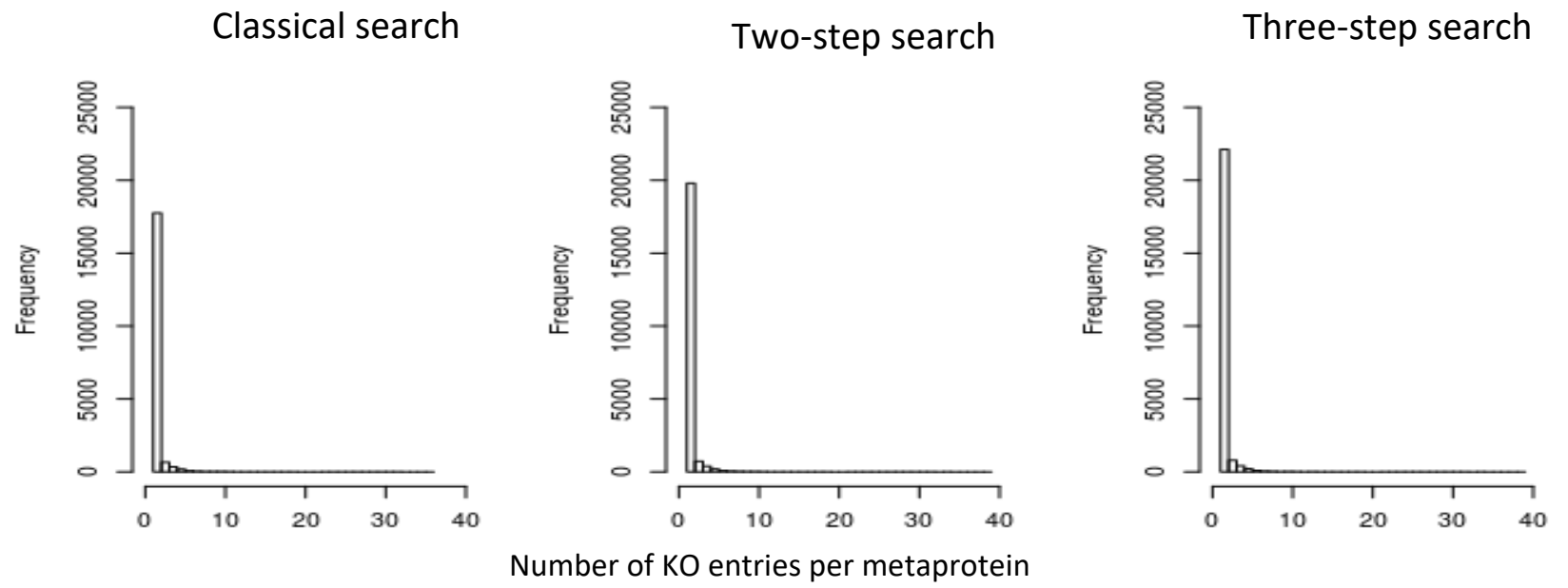

Figure S7. Distribution of metaproteins as a function of KO entries embedded: most metaproteins have onlv one $\mathrm{KO}$ entrv.

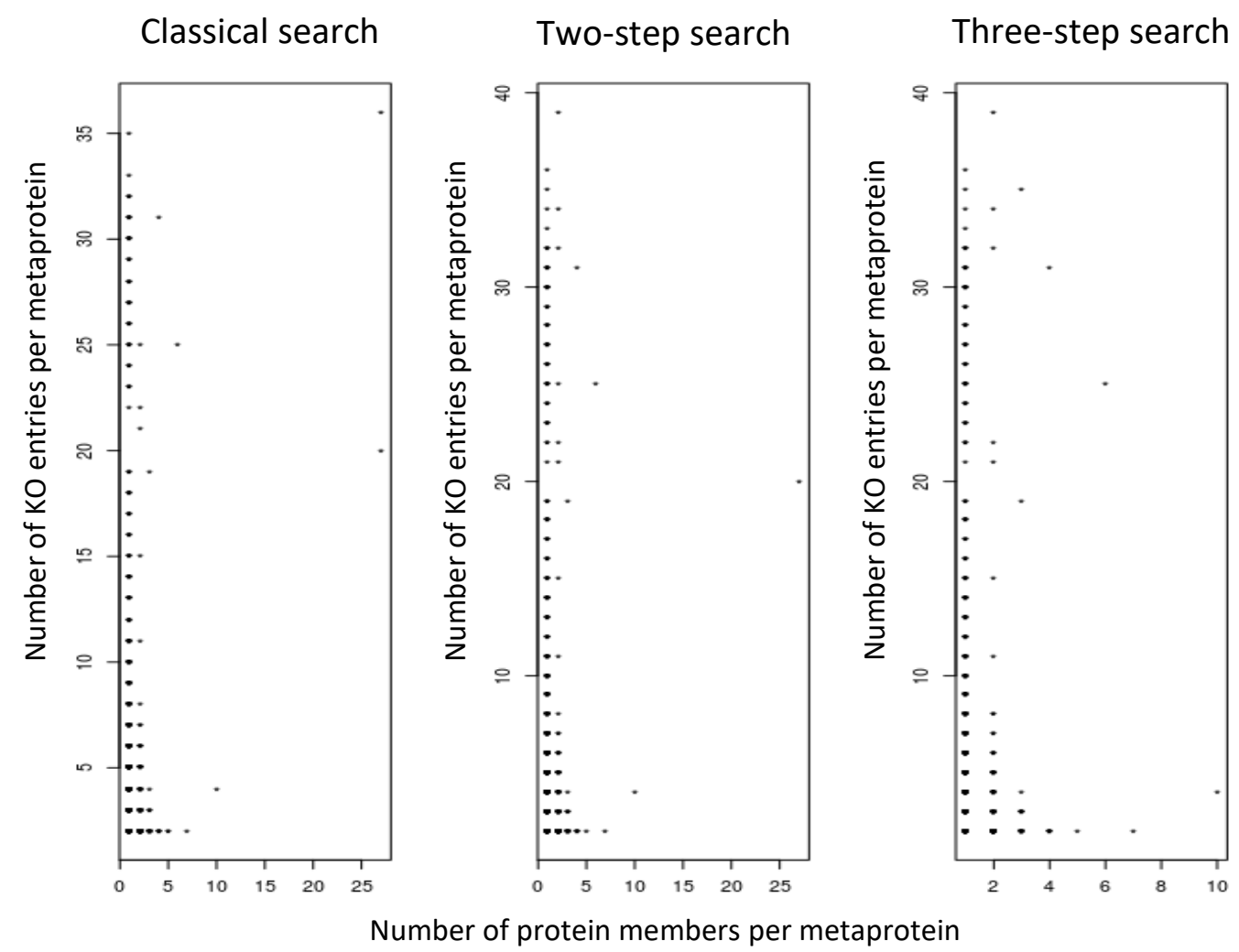

Figure S8. If present, the functional diversity of metaproteins is related to the own functional diversity of its component proteins, not to their number. 

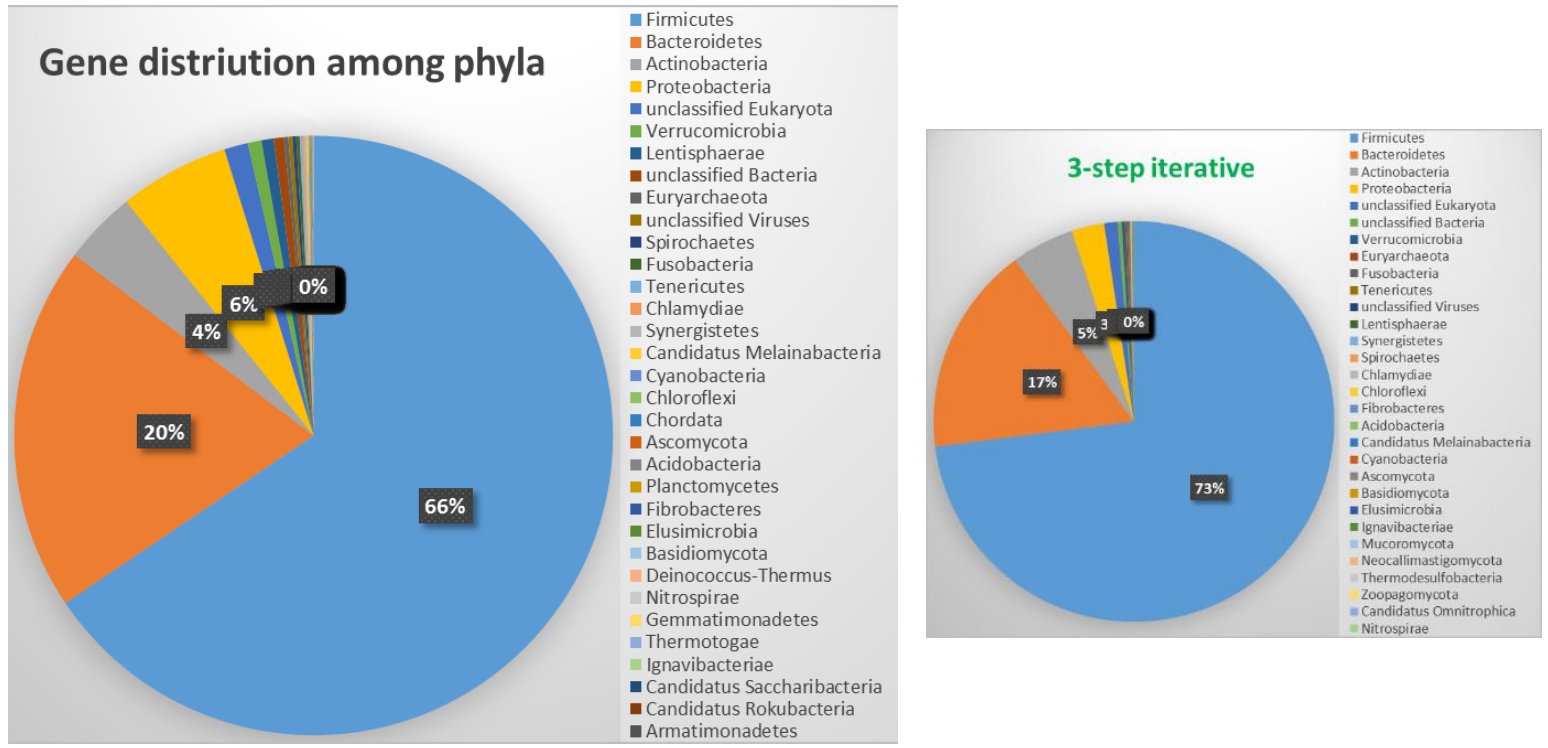

Figure S9. Percentages of genes (A) and metaproteins (B, recall of Figure 4A) assigned to the different phyla within the 48 microbiomes. 

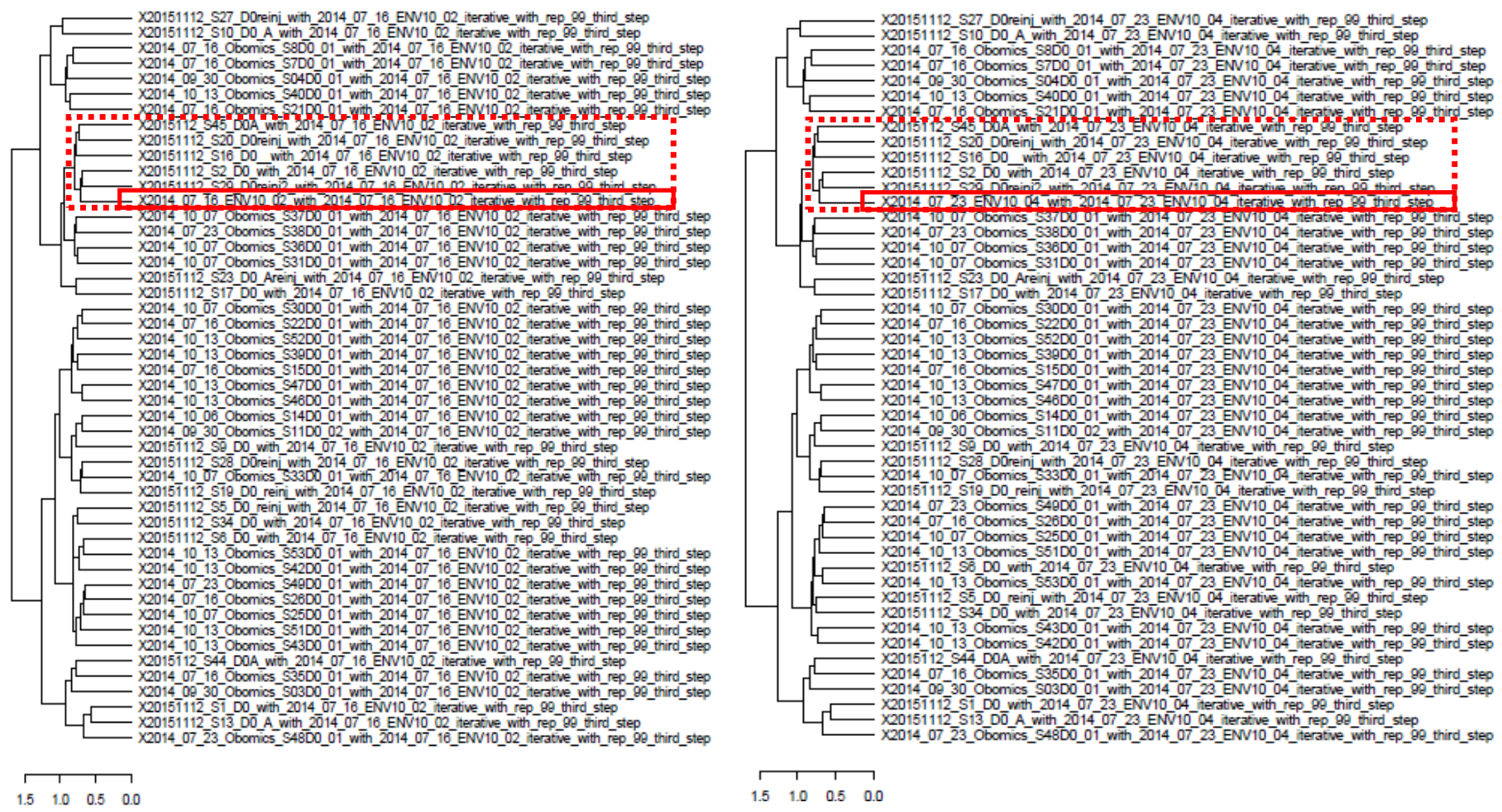

Figure S10. Example of reproducibility of the positioning of replicates relative to other samples in the cohort, based on relative abundances of all metaproteins. Here two clusterings from the three-step iterative search strategy were randomly drawn among the fourteen. The two replicates (bordered with red solid line) clusterized with the same five non-replicated samples (bordered with red dashed line). The same figure was observed for all replicates, and for all three search strategies. 


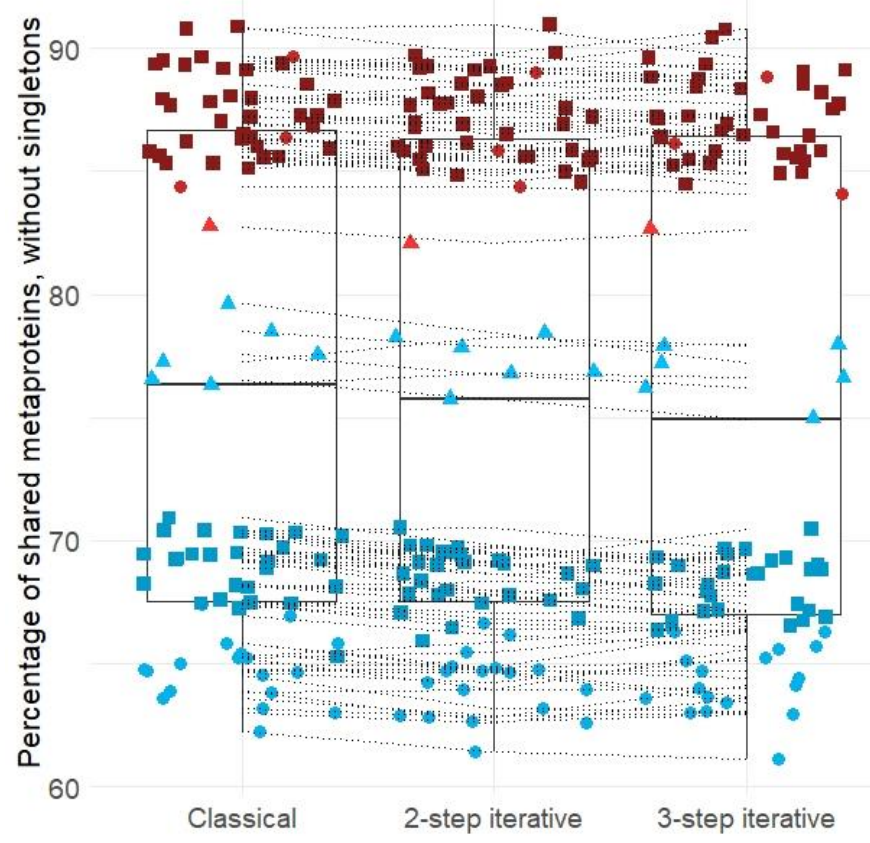

Figure S11. Metaproteins overlapping in all pairs of replicates, with each interrogation strategy. Singletons, which are metaproteins with only one specific peptide of very low abundance (one SC) in one of the two replicates only, were omitted. 